Bull. Mater. Sci., Vol. 8, No. 1, February 1986, pp. 29-38. (C) Printed in India.

\title{
Surface morphology and properties of GaAs epilayers controlled by temperature difference method of liquid phase epitaxy
}

\author{
Y K SU, C C WEI, S C LU and C C CHANG \\ Research Institute of Electronic and Electrical Engineering, National Cheng Kung Univerșity, \\ Tainan, Taiwan, Republic of China
}

MS received 25 February 1985; revised 17 September 1985

\begin{abstract}
Epilayers of gallium arsenide were grown by using the steady-state temperature difference method of liquid phase epitaxy. The surface of grown layers was smooth and shiny. Carrier concentrations of films varying from $10^{16}$ to $10^{17} \mathrm{~cm}^{-3}$ could be obtained with good reproducibility. The surface morphology growth rate, carrier concentration and Hall mobility of the epilayers were studied. Several distinct types of surface features were also investigated and explained. A segregation coefficient for the net carrier concentration versus tin concentration in the growth melt was calculated as $1.84 \times 10^{-4}$ at $700^{\circ} \mathrm{C}$ for $(100) \mathrm{GaAs}$ substrate. Thickness control for epilayers down to submicron can be obtained reproducibly.
\end{abstract}

Keywords. Liquid phase epitaxy; GaAs thin films; surface morphology.

\section{Introduction}

The use of liquid phase epitaxy to grow GaAs layers onto GaAs substrates was pioneered by Nelson (1963) using a tipping furnace. Subsequently, horizontal sliding (Mlavsky and Weinstein 1963), vertical dipping (Shih et al 1962) and rotating methods (Vilms and Garrett 1972) have been used for layer growth along with every variety of boat and slider designs. Multiple-layer structures have wide applications in the fabrication of opto-electronic devices.

Most authors in developing liquid phase epitaxial system used transient system (Su et al 1984; Wu et al 1985). In this paper, experiments were made by the steady-state LPE method. The advantages of this method are: (1) easier temperature control compared with the transient method; (2) constant growth rate of the thermal gradient and the system temperature are kept constant. The desired thickness of epilayer can be obtained by precisely controlling the growth time; (3) since there is a temperature gradient imposed normal to the growing interface, constitutional supercooling can be inhibited (Wei et al 1981). Single and double epilayers were grown onto GaAs substrate. Tin was used as the $n$-type dopant.

The growth apparatus and experimental procedure will be described in $\$ 2$. Section 3 deals with the experimental results and discussions about the surface morphology, the growth rate, and the properties of epilayers and the conclusions are presented in $\$ 4$.

\section{LPE apparatus and experimental procedure}

A sliding boat for the growth system was used in our experiment. The experimental setup consisted of a central quartz reactor tube with $54 \mathrm{~mm}$ in O.D. and $48 \mathrm{~mm}$ in I.D., a 
transparent furnace with a reflecting gold $(\sim 200 \AA)$ coated on the inner surface. This furnace had a flat temperature zone within $\pm 1{ }^{\circ} \mathrm{C}$ over $70 \%$ of its length. The graphite boat similar to that used by Dawson (1974) was made of high purity graphite (POCO Co.). It consisted of a sliding part with two wells of size $10 \times 10 \times 0.4 \mathrm{~mm}^{3}$ which were used to hold the major substrate and a precursor substrate if necessary during growth. Another part of the graphite boat contained three crucibles for melt. Two tungsten subheating wires were wound around the upper side of the melt crucibles to produce temperature difference $(\Delta T)$. An additional power of 12 watts produced a temperature difference of about $10^{\circ} \mathrm{C}$ in the melt. Two $\mathrm{Pt}-\mathrm{Pt}+13 \% \mathrm{Rh}$ thermocouples were used to measure the imposed temperature difference and control the growth temperature respectively.

After cleaning, etching and loading the substrates and sources into the furnace, it was sealed and evacuated, then backfilled with purified $\mathrm{H}_{2}$ and kept on purging with a flow rate of $0.51 / \mathrm{min}$ for $30 \mathrm{~min}$. The furnace was then heated to the growth temperature, $700^{\circ} \mathrm{C}$ and stabilized for about 1-2 hr to equilibrate and homogenize the solution. Temperature difference between substrate and sources was established by turning on the power supply. A few minutes later, the substrate was pushed into contact with the first melt, then the second one successively for a given time interval, then pushed away and the growth run terminated. The furnace was then moved away from the boat. The substrate was removed from the furnace after cooling.

The surface morphology of epilayers was examined with a Nomarski phase contrast microscope. Thickness was measured with optical microscope by suitably staining the cleavage with MSO photo-etchant (Wei et al 1981). Van der Pauw-Hall measurements were performed to calculate the electrical properties of the epilayers. Doping concentration and uniformity could be deduced from $\mathrm{C}-\mathrm{V}$ measurements. In order to do this, Al Schottky barriers of $200 \mu \mathrm{m}$ in diameter had been evaporated through a photomask.

\section{Results and discussion}

\subsection{Surface morphology}

There are several distinct types of surface features: (1) meniscus lines, (2) cusps, (3) island growth, (4) terraces and (5) nearly perfect flat. The meniscus lines are shown in figures 1(a) and (b). It has been proved that these lines are instantaneous trailing edge of the liquid surface at it moved across the crystal during the sliding procedure (Small et al 1975). They are roughly parallel and spaced about $100+50 \mu \mathrm{m}$ apart with height about $100 \AA$. Device fabrication is not seriousiy perturbed if we fabricate them parallel to the meniscus lines.

Figure 1(b) also shows the cusps. The formation of cusps neither corresponds to dislocations in the substrate nor corresponds to contaminating particles on the substrate before growth. The factors governing this surface morphology are still not understood. The density of the cusps is about $1 \cdot 1 \times 10^{3} / \mathrm{cm}^{2}$.

Figure 2(a) shows the pattern of growth on a (100) substrate where the solution was about $20^{\circ} \mathrm{C}$ below liquidus temperature. The steps or "mesas" appeared to be caused by the spotty contact between the solution and the substrate because of severe supersaturation. The top of the mesa forms a rectangle whose sides are parallel to the 


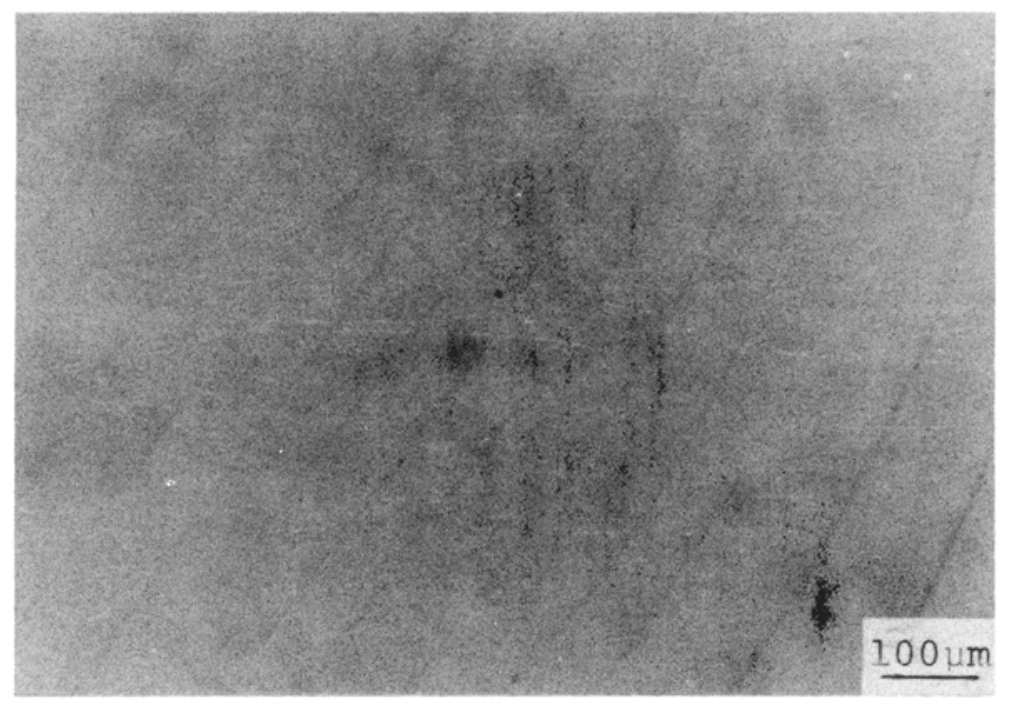

(a)

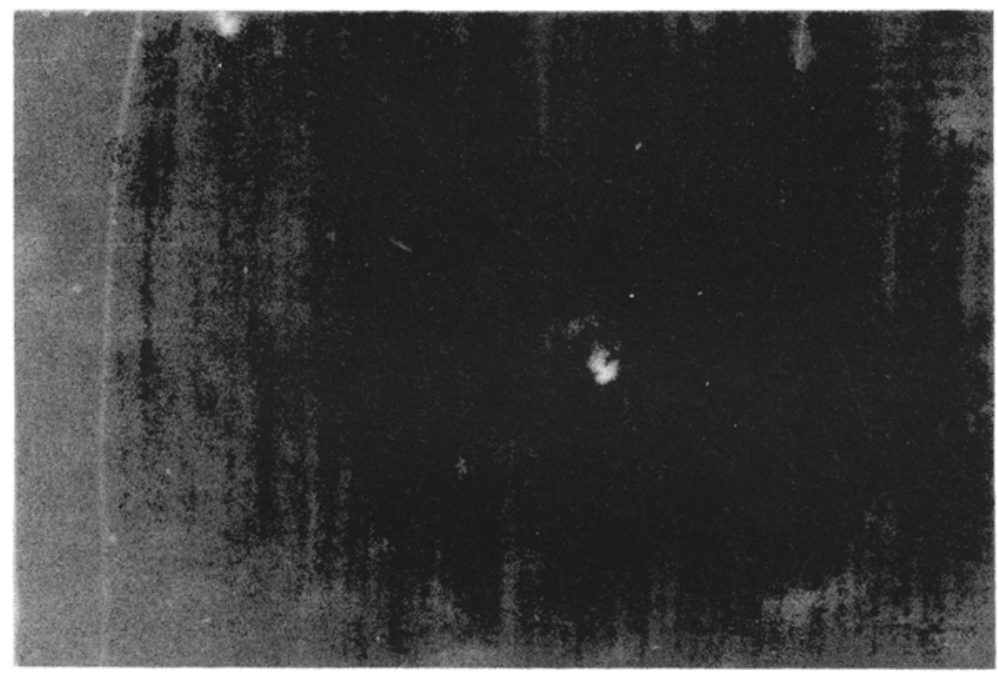

(b)

Figure 1. (a) Optical micrograph showing meniscus lines. (b) Nomarski contrast micrograph showing meniscus line and cusps.

[110] axis. The trapezoidal planes which form the sides of the mesa line in the (111) plane can be shown in the cleaved edge view of figures 2(a) and 2(b).

Another kind of surface morphology is terraces as shown in figure 3(a). Mattes and Route (1974) have found that the surface terraces appear to be influenced by the nuclei formations and thermal fluctuations at the liquid-solid interface. Figure 3 (b) shows the cleaved and stained edge view of figure 3(a). From this figure we can find that the terraces look like a triangular waveform, the "peak to peak" height is about $1.2 \mu \mathrm{m}$, too severe to be used for device fabrication. 

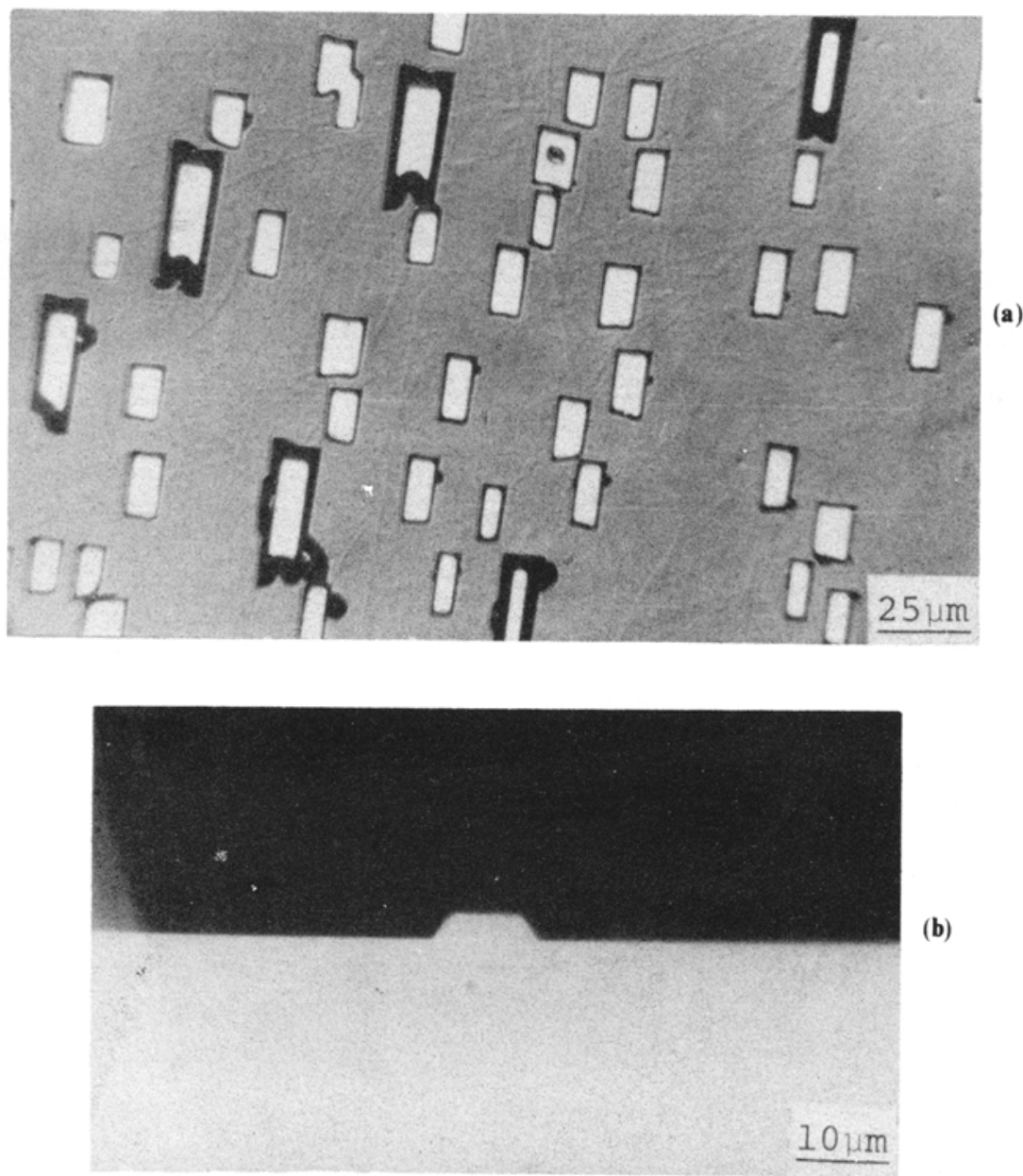

(b)

Figure 2. (a) Island growth of LPE on a (100) substrate. (b) Higher magnification cleaved edge view of (a).

The last type of feature, nearly perfectly flat regions with no structure observable by Nomarski phase contrast microscope is shown in figure 4 . This smooth and mirror-like epilayers can be reproducibly obtained for the optimum growth condition.

\subsection{Growth rate}

Thickness control for layer down to submicron is obtained by properly controlling the temperature difference and cooling rate. Any residual Ga melt left on the surface of epilayer will cause an extra growth of 5 to $10 \mu \mathrm{m}$ during the cooling interval and cannot be used for the fabrication of devices. Removing the growing epilayer from the solution with a rapid and smooth motion helps to minimize the adherence of liquid Ga droplets on the surface of epilayer.

The dependence of epilayer thickness on the growth time for $\Delta T=5^{\circ} \mathrm{C}$ and $10^{\circ} \mathrm{C}$ is shown in figure 5. The growth temperature is kept at $700^{\circ} \mathrm{C}$ throughout our 


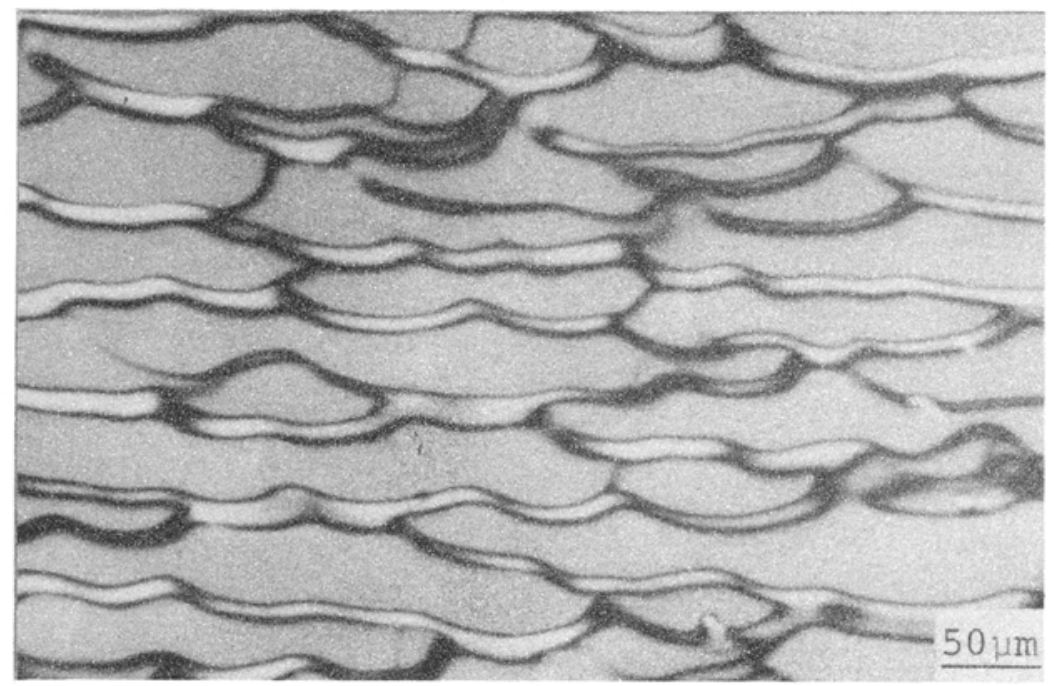

(a)

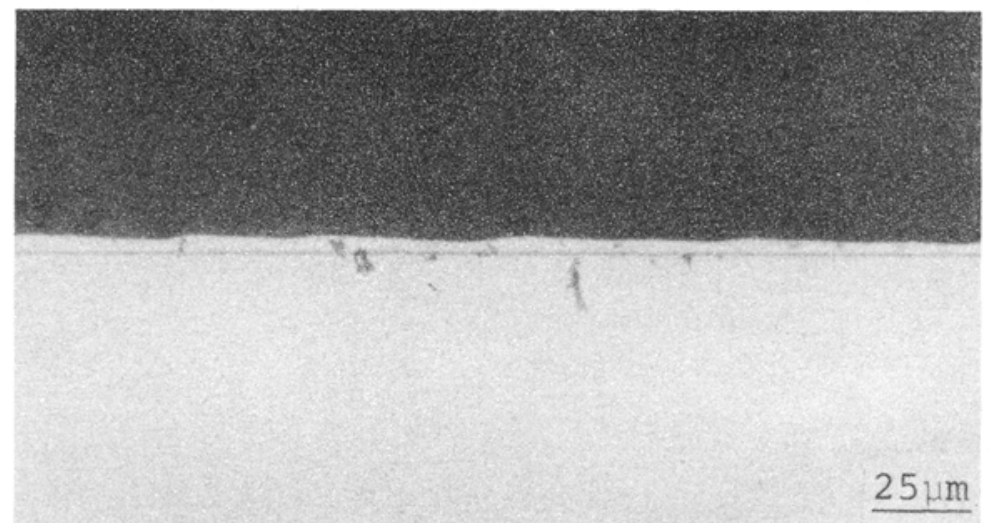

(b)

Figure 3. (a) Nomarski contrast micrograph of epilayers showing terraces. (b) The cleaved and stained edge view of (a).

experiments. Temperature control is carried out by the main heater wound on the reaction tube mentioned above, while the substrates are used to impose temperature difference $(\Delta T)$. The layer thickness is proportional to the growth time for two different $\Delta T$, and these results are similar to those obtained by Nishizawa and Okuno (1978). The growth rates are about $0.1 \mu \mathrm{m} / \mathrm{min}$ and $0.22 \mu \mathrm{m} / \mathrm{min}$ for $\Delta T=5^{\circ} \mathrm{C}$ and $10^{\circ} \mathrm{C}$ respectively.

All the layer thickness are determined by cleaving (110) surface, then staining and measuring by optical microscope. This measurement results are also illustrated in figure 6 which shows the cross-sectional view of some single and double epilayers.

\subsection{Doping study with tin}

Tin is selected as $n$-type dopant in spite of its amphoteric property because of its extremely low vapour pressure, low segregation coefficient under the epitaxial growth 

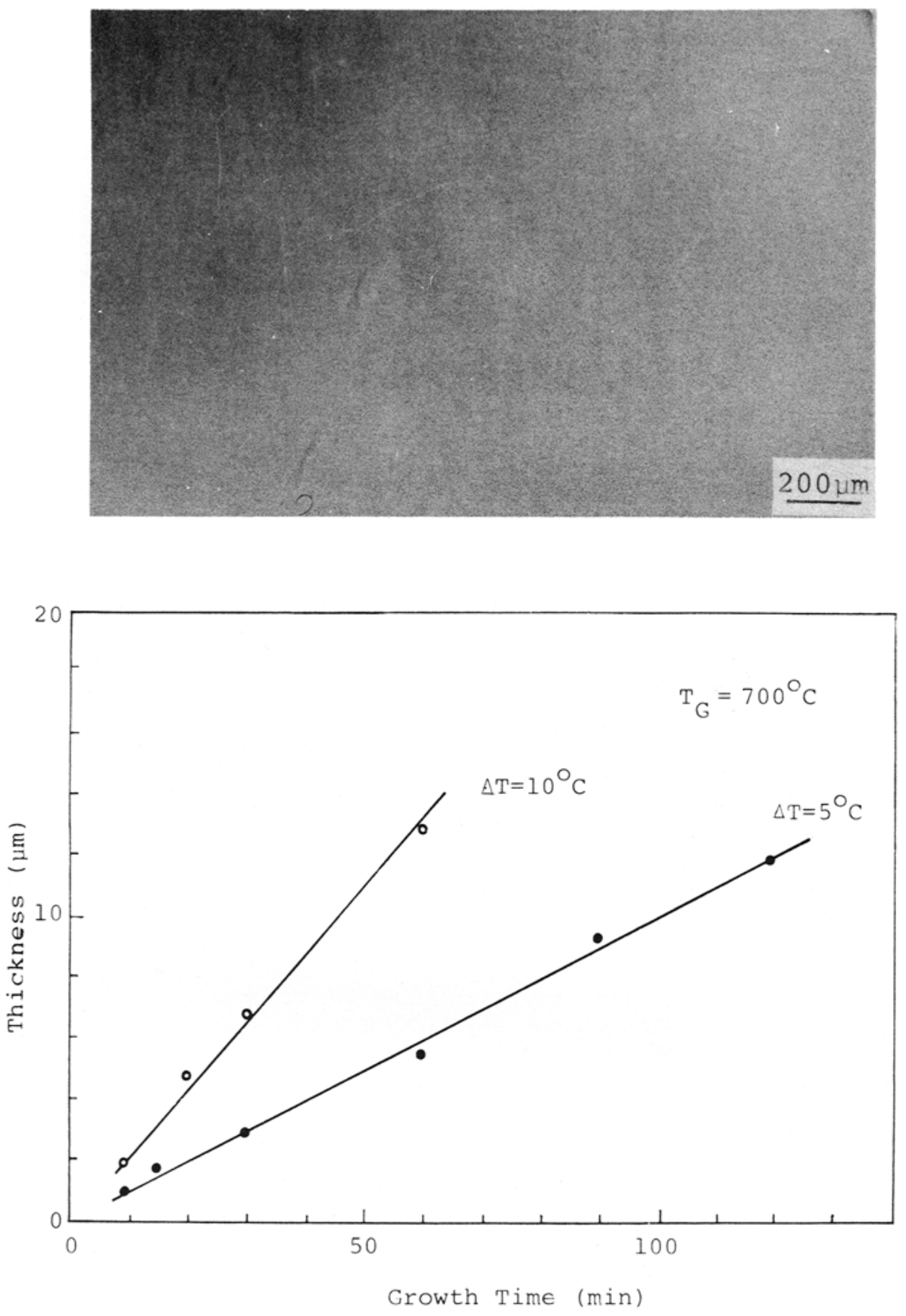

Figure 5. Epilayer thickness as a function of growth time for $\Delta T=10^{\circ} \mathrm{C}$ and $\Delta T=5^{\circ} \mathrm{C}$.

condition and solubility in gallium (Shunt 1969). Figure 7 shows the carrier concentration of epitaxial films as a function of tin mole percent in the growth solution at $700^{\circ} \mathrm{C}$. Based on our data, the segregation coefficient for GaAs growth from $\mathrm{Ga}$ solution at $700^{\circ} \mathrm{C}$ is $1.84 \times 10^{-4}$. This is in good agreement with the values reported 


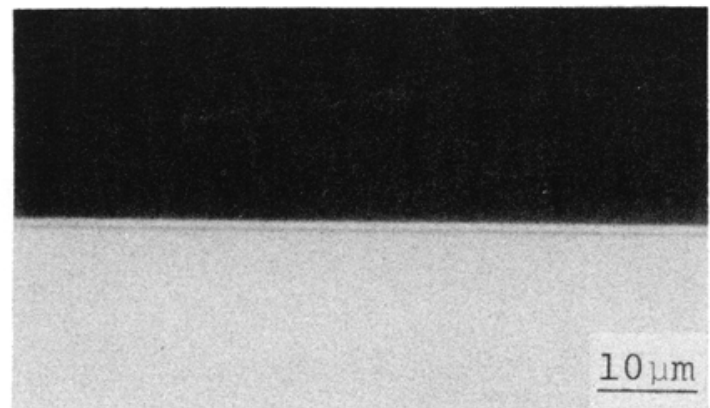

(a)

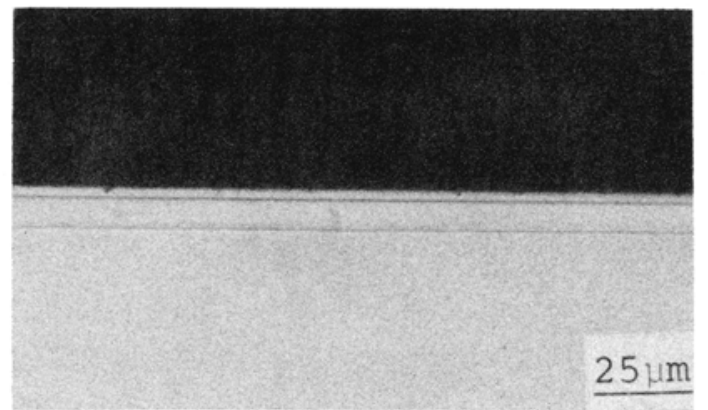

(b)

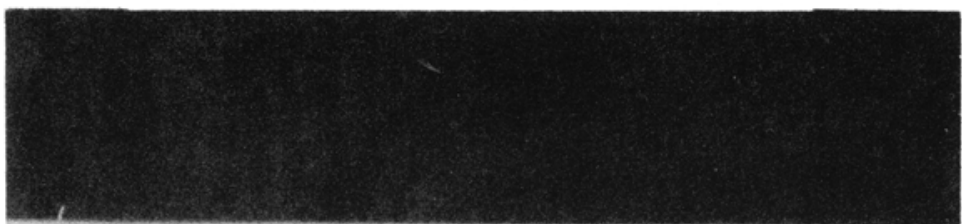

(c)

\section{$10 \mu \mathrm{m}$}

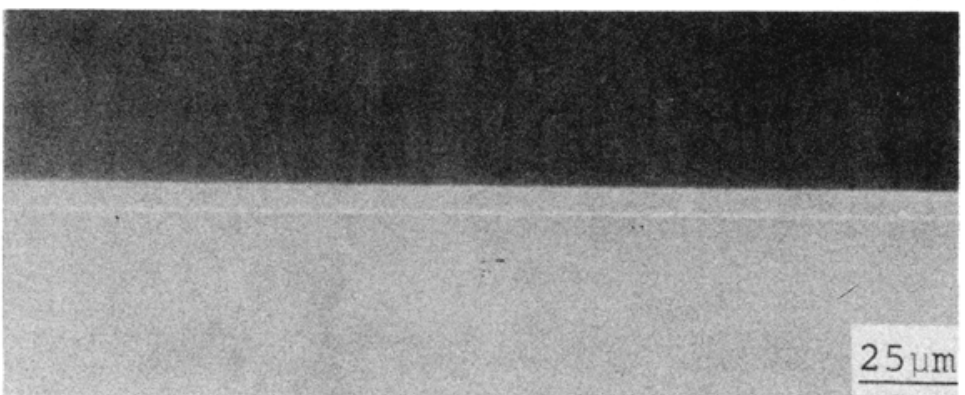

(d)

Figure 6. Cleaved and stained cross-sectional view of epilayers. (a) Single layer, $1 \mu \mathrm{m}$; (b) Double layer, $2.5-7.5 \mu \mathrm{m}$; (c) Single layer, $0.5 \mu \mathrm{m}$; (d) Double layer, $0.3-2.3 \mu \mathrm{m}$. 


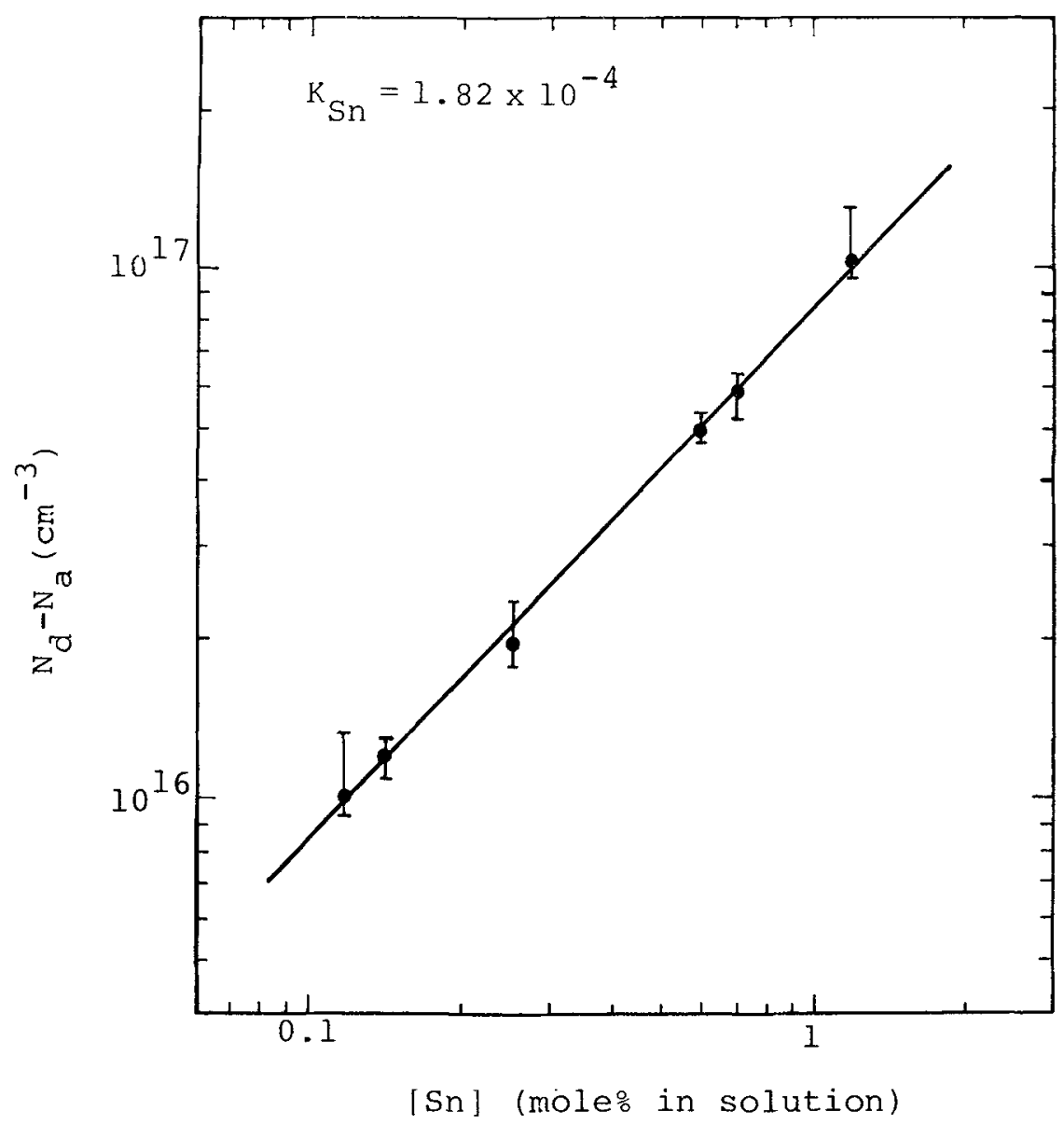

Figure 7. Carrier concentration of grown layers as a function of tin mole percent in growth solution at $700^{\circ} \mathrm{C}$.

elsewhere (Harris and Synder 1969; Vilms and Garrett 1972; Rosztoczy and Kinoshita 1974; Toyada et al 1976).

In order to measure the Hall mobility, a series of epilayers are grown from growth melt with increasing amount of $\mathrm{Sn}$ on semi-insulating (100) oriented substrates. The Hall mobility at room temperature as a function of carrier concentration is shown in figure 8.

The $\mathrm{C}-\mathrm{V}$ measurement is plotted in figure 9, indicating that the doping concentration is extremely uniform and $N_{D}$ is equal to $2.16 \times 10^{16} / \mathrm{cm}^{3}$. A built-in voltage of 0.7 volts is obtained from the intercept of the voltage axis.

\subsection{X-ray diffraction}

$\mathrm{X}$-ray diffraction method is utilized to determine the orientation of the GaAs epilayer on the GaAs substrate. The peak of the spectrum represents the (200) reflection of GaAs. This indicates that the epilayer has good crystallinity properties and the orientation of the epilayer is the same as that of the substrate. 
Surface morphology and properties of GaAs epilayers

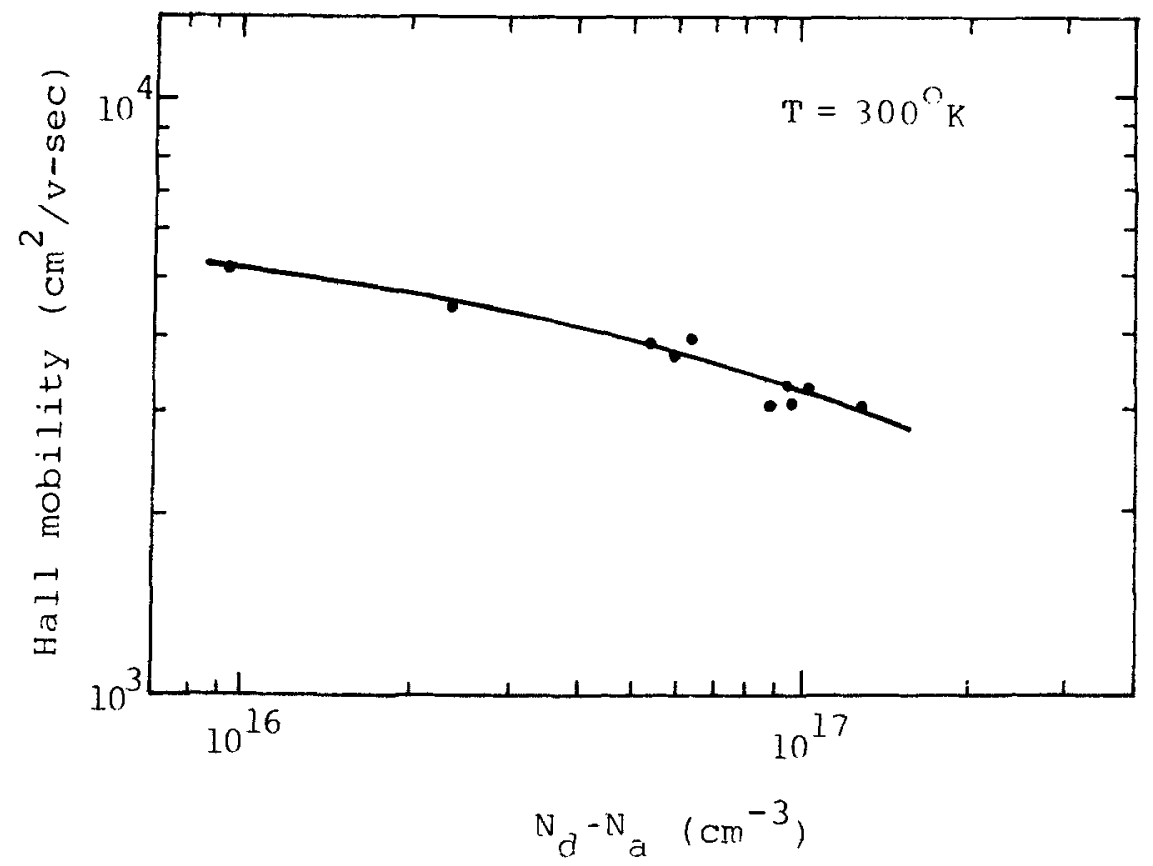

Figure 8. Hall mobility of Sn-doped n-type GaAs layer versus carrier concentration at $300^{\circ} \mathrm{K}$.

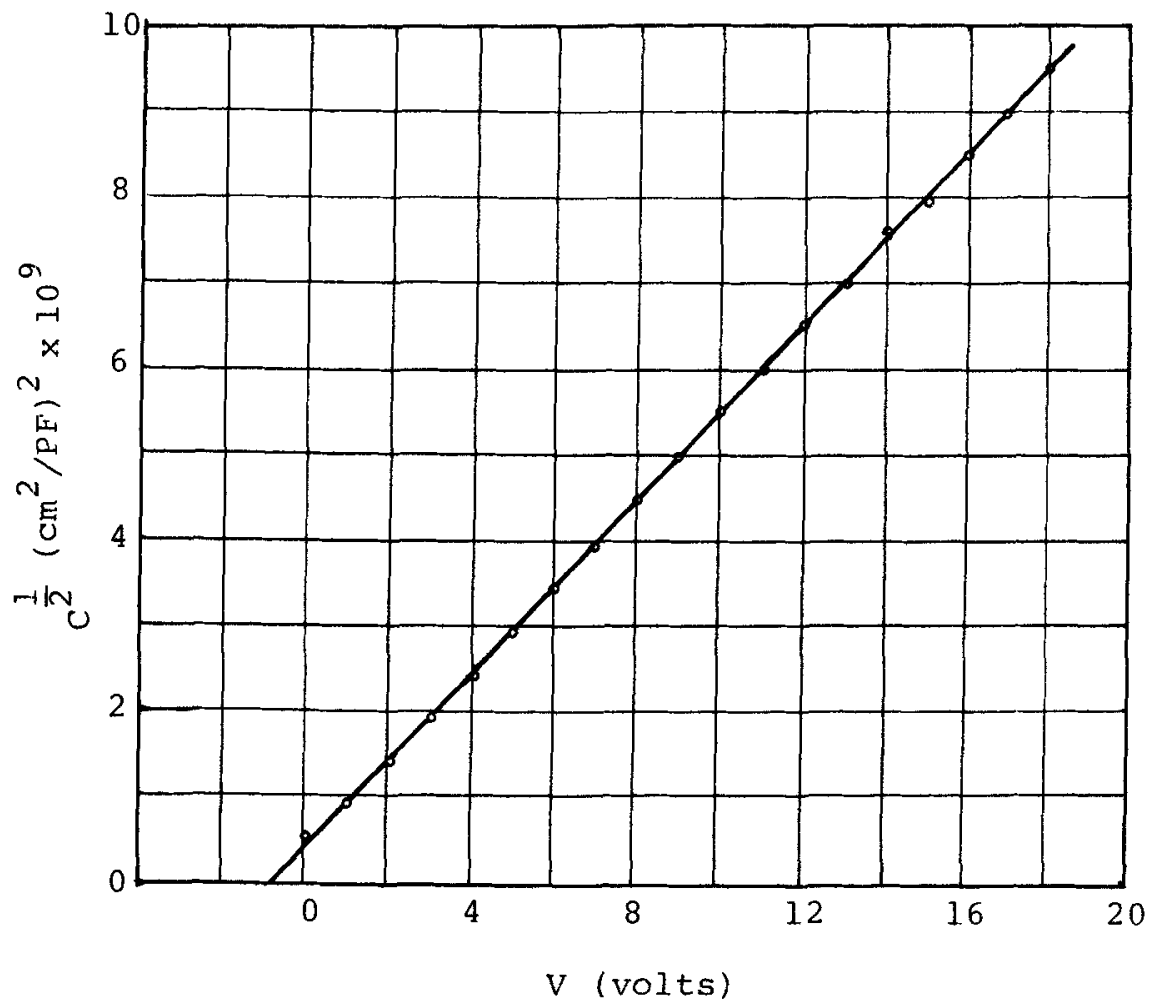

Figare 9. $1 / C^{2}$ versus applied voltage for the Schottky diode indicating $N_{D}$ is a constant. 


\section{Conclusion}

Epilayers of gallium arsenide were grown by using the temperature difference method of liquid phase epitaxy. The thickness of thin films can be precisely controlled even down to submicron meter. Also, doping concentration can be varied by the amount of tin added into the growth solution and the results showed that the uniformity of doping is very good. The carrier concentration ranges from $10^{16}$ to $10^{17} \mathrm{~cm}^{-3}$. X-ray diffraction shows that the epilayers have good crystalline properties and have the same orientation as the substrate. Several distinct types of surface features are also investigated. The segregation coefficient for the net carrier concentration versus tin concentration in the melt is calculated as $1.84 \times 10^{-4}$ at $700^{\circ} \mathrm{C}$ for $(100) \mathrm{GaAs}$ substrate.

\section{Acknowledgements}

The authors gratefully acknowledge $\mathrm{Dr} T \mathrm{~T} \mathrm{Wu}$ for his fruitful discussions and suggestions. The authors also thank the National Science Council, Republic of China for financial support in this research project (NSC 75-0608-E006-05R).

\section{References}

Dawson L R 1974 J. Cryst. Growth 2786

Harris J S and Synder W L 1969 Solid State Electron 12337

Mattes B L and Route R K 1974 J. Cryst. Growth 27133

Mlavsky A I and Weinstein M $1963 \mathrm{~J}$. Appl. Phys. 342885

Nelson H 1963 RCA Rev. 24603

Nishizawa J and Okuno Y 1978 RIEC Technical Report, TR-41 Apr.

Rosztoczy F E and Kinoshita J 1974 J. Electrochem. Soc. 121439

Shih K K, Woodall J M, Blum S E and Foster L M 1968 J. Appl. Phys. 392962

Shunt F A 1969 Constitution of binary alloys (New York: McGraw-Hill) 2nd Suppl.

Small M B, Blakeslee A E, Shih K K and Potemski R M 1975 J. Cryst. Growth 30257

Su Y K, Wu M C, Cheng K Y and Chang C Y 1984 J. Cryst. Growth 67477

Toyada N, Mibara M and Hara T 1976 J. Appl. Phys. 47443

Vilms J and Garrett J P 1972 Solid State Electron 15443

Wei C C, Su Y K, Chang C C, Liu B D and Lu S C 1981 Chinese J. Mater. Sci. 1356

Wu M C, Su Y K, Chang C Y and Cheng K Y 1985 J. Appl. Phys. (to be published) 